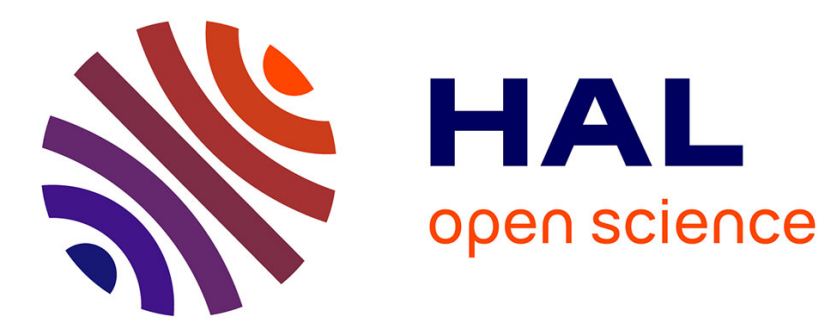

\title{
Quel urbanisme face aux mutations de la société postindustrielle?
}

\author{
Albert Levy
}

\section{To cite this version:}

Albert Levy. Quel urbanisme face aux mutations de la société postindustrielle?. Revue Esprit, 2006, pp.61-75. halshs-00120458

\section{HAL Id: halshs-00120458 \\ https://shs.hal.science/halshs-00120458}

Submitted on 20 Feb 2007

HAL is a multi-disciplinary open access archive for the deposit and dissemination of scientific research documents, whether they are published or not. The documents may come from teaching and research institutions in France or abroad, or from public or private research centers.
L'archive ouverte pluridisciplinaire HAL, est destinée au dépôt et à la diffusion de documents scientifiques de niveau recherche, publiés ou non, émanant des établissements d'enseignement et de recherche français ou étrangers, des laboratoires publics ou privés. 


\title{
Quel urbanisme face aux mutations de la société post-industrielle? Vers un urbanisme transactionnel.
}

\begin{abstract}
Albert Levy*
Où va l'urbanisme en France? Comment évolue sa pratique? Quelles transformations le nouveau contexte idéologique et économique de la société post-industrielle a-t-il induit sur la ville, sa forme et son mode de production ? A quels problèmes urbains doit-il faire face aujourd'hui et quelles solutions nouvelles a-t-il imaginé ? Quels discours urbanistiques innovants a-t-il produit? Que vaut la notion de projet urbain ? Notion floue, générique et polysémique, elle a envahi la pratique urbanistique et semble recouvrir indifféremment toutes les tendances urbanistiques sans distinction. On essayera de répondre à ces questions en analysant les transformations actuelles du contexte de production de l'urbain pour comprendre leur impact sur l'urbanisme et son évolution, en nous appuyant sur un exemple parisien, le projet urbain Paris Rive Gauche, un projet qui se présente comme un modèle innovant dans ce domaine.

Situé à l'Est de Paris, en bordure de la Seine, sur la rive gauche, couvrant 130 hectares, sur de $2,7 \mathrm{~km}$ de long, le projet Paris Rive Gauche (PRG) est la plus importante opération d'urbanisme en cours dans la capitale. C'est un projet de renouvellement urbain et de requalification d'une friche ferroviaire et industrielle, autour et à partir de la gare d'Austerlitz, qui remonte à 1965, date du Schéma directeur régional, et à 1973 avec le Schéma de secteur Seine Sud-Est, premiers plans élaborés à la suite des effets ravageurs de la crise de désindustrialisation qu'ont connu Paris et la région parisienne. Trois événements ont marqué le déroulement de cette opération de renouvellement : 1'arrivée de la BNF en 1989, véritable date de départ de la ZAC PRG; l'introduction de la loi Lepage en 1997 qui installe la concertation ; le changement de majorité municipale en 2001 qui amène une majorité de gauche à la mairie de Paris, entraînant un fléchissement du projet, de sa méthode et de son programme. Ce projet offre un cas intéressant d'étude pour illustrer ce nouvel urbanisme émergent que nous avons proposé d'appeler urbanisme transactionnel.
\end{abstract}

\section{Quels nouveaux discours en urbanisme?}

L'urbanisme est né en Occident avec la révolution industrielle. En passant de l'art urbain à l'urbanisme ${ }^{1}$, la " discipline » nouvelle voulait apporter des solutions, " scientifiquement » fondées, aux bouleversements engendrés par l'industrialisation et à ses conséquences sur le plan social et urbain, apporter des remèdes aux maux causés par les dysfonctionnements du système économique capitaliste et ses répercussions néfastes sur la ville. Dans sa réponse, l'urbanisme s'est subdivisé en plusieurs courants idéologiques opposés, progressiste, culturaliste, naturaliste ${ }^{2}$. Avec les mutations socio-économiques et technologiques de ces dernières décennies, l'accélération et l'amplification de la mobilité, l'explosion des technologies informationnelles, l'accentuation des inégalités et des ségrégations ${ }^{3}$, le processus de métropolisation lié à la mondialisation ${ }^{4} .$. , la ville de l'ère industrielle a été totalement dépassée, dans sa forme comme dans son fonctionnement. Ses outils traditionnels

\footnotetext{
${ }^{1}$ F. Choay, Urbanisme utopies et réalités, Paris, Le Seuil, 1965

2 Ibid.

${ }^{3}$ Sur cette question, J. Donzelot, C. Mevel, A. Wyvekens, Faire société. La politique de la ville aux Etats-Unis et en France, Paris, Le Seuil, 2003.

${ }^{4}$ O. Mongin, La condition urbaine. La ville à l'heure de la mondialisation, Paris, Le Seuil, 2005
} 
d'aménagement (planification) sont devenus complètement obsolètes ${ }^{5}$, son organisation spatiale taylorienne et sa segmentation rigide définitivement révolues ${ }^{6}$.

Une économie tertiaire tournée vers les services, l'information, s'est développée, et on parle même, avec l'essor de la culture, du loisir, et du tourisme urbain..., d'une économie quaternaire. Ces transformations socio-économiques du système productif ne sont pas sans conséquences sur la ville, elles en modifient l'espace et l'usage, les échelles et les territoires. Nous sommes entrés dans un nouvel age urbain, l'age post-industriel ${ }^{7}$, qui correspond à une nouvelle révolution économique avec un nouveau type de ville: des problèmes inédits émergent qui attendent des solutions nouvelles. La production de richesse devenue surtout immatérielle, c'est, à présent, la mobilisation du capital humain qu'exige le nouveau système productif. L'économie urbaine, et la valeur, ne peuvent plus être réduites au simple capital physique et matériel, de même que la définition du patrimoine ne peut plus être limitée au bâti historique uniquement (tissus anciens et monuments) : outre ce patrimoine historique, doivent être également pris en compte le patrimoine humain (éducation, santé, culture,...), scientifique (savoirs et savoir-faire, structures universitaires, centres de recherche...), environnemental (climat, nature, paysage...), l'ensemble des infrastructures, équipements et services, spécifiques à une ville, accumulés à travers son histoire et constitutifs de son potentiel productif. Dans cette nouvelle acception du patrimoine, la notion de valeur et les éléments pris en considération sont donc élargis, ils permettent de mieux comprendre la nature de système économique post-industriel actuel et l'espace métropolitain où il s'inscrit.

Face à ces mutations socio-économiques où domine à présent la production de richesses surtout immatérielles, face à cette ville post-industrielle et à ses problèmes, assiste-t-on à une nouvelle génération de discours urbanistiques comparables à ceux de l'époque précédente qui a donné naissance à l'urbanisme ? Ce qu'on appelle, par exemple, actuellement, " urbanisme postmoderne », " renouvellement urbain », « ville durable », " Grand Projet de Ville » (GPV dans le cadre de la politique de la ville)... ces courants s'appuient-ils sur la construction de discours urbanistiques cohérents ? Constituent-t-ils des théorisations doctrinales explicites et formalisées comme celles de la précédente génération? Apportent-ils des solutions aux nouvelles questions posées?

En suivant la typologie des discours de F. Choay, on retrouverait encore de nos jours les trois types discursifs urbanistiques principaux (progressiste/culturaliste/naturaliste) qu'elle avait bien cernés, et dont elle avait aussi pressenti le prolongement à travers les notions de 《technotopie » (ou «technolâtrie », le salut par recours à la technique ${ }^{8}$ ), « anthropopolis » (critique humaniste basée sur les sciences sociales valorisant le passé, la continuité, la tradition, les formes urbaines anciennes ${ }^{9}$ ), et «philosophie anti-urbaine» (discours critiques

\footnotetext{
${ }^{5}$ La loi SRU (Solidarité et Renouvellement Urbain, 2000) a voulu actualiser ces outils d'aménagement périmés.

${ }^{6} \mathrm{P}$. Veltz, Mondialisation, villes et territoires, Paris, Puf, 2005

${ }^{7}$ Certains ont parlé d'age post-urbain (M. Webber, The Post-city age, Daedalus, Fall, 1967 ; F. Choay, Préface à M. Webber, L'urbain sans lieu ni borne, La Tour d'Aigues, Ed. de l'Aube, 1996), ou fin des villes, mais la réalité a démenti ses prophéties et on assiste plutôt, avec la métropolisation, à une nouvelle forme de concentration urbaine, là-dessus, P. Veltz, op. cit. D. Cohen, Trois leçons sur la société post-industrielle, Paris, Le Seuil, 2006. L'auteur voit cinq ruptures majeures: une troisième révolution industrielle (la révolution informatique après la machine à vapeur et l'électricité), une nouvelle conception du travail (fin du fordisme), l'essor de l'individualisme (mai 68), l'explosion des marchés financiers, la mondialisation des échanges.

${ }^{8}$ Elle cite comme exemples : E. Hénard, Buchanan, I. Xenakis, et les auteurs des villes futuristes, L'urbanisme, ... op.cit.

${ }_{9}$ Elle donne comme représentants de ce groupe : P. Geddes, M. Poète, L. Mumford, J. Jacobs, L. Duhl, K. Lynch, Ibid.
} 
contre la ville décadente et retour à la nature salvatrice ${ }^{10}$ ). Cette typologie se poursuit aujourd'hui. Le progressisme connaît un nouvel avatar sous le terme d'hypermodernité ${ }^{11}$ (ou surmodernité): on y retrouve la même idéologie de contrôle de l'espace urbain par la technoscience dominante. Le culturalisme se prolonge dans le postmodernisme ${ }^{12}$ avec la même critique de l'illusion progressiste et de son optimisme, et une même attitude nostalgique vis-à-vis de la ville du passé et de ses formes. Quant à la philosophie anti-urbaine, elle est présente dans certains discours écologistes ${ }^{13}$ qui survalorisent la nature, mettant en cause l'extension inhumaine des métropoles destructrices de l'environnement, constituant une menace pour l'humanité.

Aucune de ces versions, cependant, n'a donné lieu à des constructions discursives doctrinales, cohérentes, explicitement formulées (sinon peut-être pour la « ville durable » ${ }^{14}$ ) comparables aux précédentes formulations doctrinales de l'ère industrielle. La persistance de cette typologie des discours, à travers ces nouveaux avatars, avec leur absence d'explicitation, leur faible formalisation, sont encore des hypothèses de travail à approfondir. Elle soulève une autre question importante : ces "discours" sont-ils appropriés aux problèmes actuels de la ville post-industrielle, sont-ils des réponses adéquates aux conséquences de la métropolisation, au contexte de la mondialisation et de son impact?

Outre ce premier aspect, le vide doctrinal actuel de l'urbanisme et l'absence de modèles urbains cohérents qui pensent le rapport ville et société post-industrielle, engendrant une dispersion des idées et des positions éclectiques, trois autres aspects caractérisent la situation contemporaine dans laquelle évolue l'urbanisme actuellement qui voit apparaître une nouvelle notion, le projet urbain : la formation d'une nouvelle culture du projet ; la complexification du système d'acteurs avec émergence de nouvelles fonctions dans l'aménagement ; l'irruption et l'essor du débat public. Ces transformations du contexte de production et des conditions d'exercice du projet ne seront pas sans conséquence sur l'évolution de l'urbanisme.

\footnotetext{
${ }^{10}$ Comme figures de ce dernier courant, elle cite : V. Hugo, G. Simmel, et surtout les philosophes O. Spengler, M. Heidegger, Ibid.

${ }^{11}$ La généalogie de ce nouvel avatar du progressisme - où la mobilité, la vitesse, et la télécommunication jouent un rôle clé - reste à faire, depuis G. Dupuy, L'urbanisme des réseaux, Paris, A. Colin, 1991 ; G. Dubois Taine, Y. Chalas, La ville émergente, La Tour d'Aigues, l'Aube, 1993 ; F. Ascher, Metapolis ou l'avenir des villes, Paris, Odile Jacob, 1995 ; Les nouveaux principes de l'urbanisme, La fin de villes n'est pas à l'ordre du jour, La Tour d'Aigues, 1'Aube, 2001... qui célèbrent les temps hypermodernes, ainsi que de nombreux autres chercheurs qui se penchent sur cette évolution de la modernité : M. Augé, Non-lieux, Introduction à une anthropologie de la surmodernité, Paris, la Librairie du siècle, 1995 ; G. Lipowetsky, Ch. Sebastien, Les temps hypermodernes, Paris, Grasset, 2004 ...

${ }^{12}$ N. Ellin, Postmodern urbanism, Cambridge Massachusetts, Blackwell Publishers, 1996, offre un bon aperçu de cette tendance.

${ }^{13}$ Voir par exemple, F. Lapoix, Sauver la ville, Ecologie du milieu urbain, Paris, Sang de la Terre, 1991 ; E. Dorier-Apprill (éd), Ville et environnement, Sedes, 2006 ; F. Beaucire, Contrainte écologique et développement urbain durable, in $\mathrm{Ph}$. Genestier (éd), Vers un nouvel urbanisme, Paris, La Documentation Française, 1996...

${ }^{14}$ Cette « ville durable », ville compacte, est évidemment aux antipodes du modèle naturaliste américain de $\mathrm{F}$. L. Wright, Broadacre city (1932), qui prônait une dispersion et une dilution de la ville dans la nature, impliquant un usage intensif de l'automobile et l'habitat individuel comme unique type de logement. Les ouvrages sur la « ville durable» et son rapport au développement soutenable (Kyoto) prolifèrent. Cette question est au centre des préoccupations internationales, on peut citer quelques grandes étapes : Commission Brundland 1987, Déclaration de Rio 1992, Charte d'Aalborg 1994, Lisbonne 1996, Hanovre 2000, Aalborg 2004 qui aboutit à une charte considérée comme texte de référence de la ville durable européenne. Les Chinois, de leur coté, prévoient la construction de 400 villes durables à l'horizon 2020, la première Dongton (500.000 hab.) devrait être terminée en 2010 par l'agence anglaise Arup.
} 


\section{Une nouvelle culture du projet}

Le premier aspect concerne la mutation et la diffusion de la notion de projet. J. P. Boutinet ${ }^{15}$ a analysé d'un point de vue anthropologique les transformations de cette notion. Outre l'élaboration d'une typologie en projet individuel/ projet d'objet/ projet d'action/ projet organisationnel/ projet de société, l'auteur propose également une lecture de l'évolution de la notion dans le temps qui débouche aujourd'hui, selon lui, sur une nouvelle culture du projet.

L'idée de projet apparaît à la Renaissance, comme innovation technique et culturelle. $\mathrm{F}$. Choay ${ }^{16}$ en a identifié et analysé les deux principales modalités opposées : la règle, c'est-àdire le projet ouvert et flexible (Alberti), et le modèle, ou le projet fermé et figé, propre à l'utopie (T. More). Elle a examiné la postérité de ce paradigme dans le temps. Au XVIIIe siècle, avec les Lumières, surgit l'idée de projet de société ${ }^{17}$, les débuts de la révolution industrielle donneront lieu aussi à un foisonnement de projets sociaux et existentiels, à caractère surtout utopique (Fourrier, Owen, Cabet, Gaudin...). Avec la société industrielle émerge le projet moderne, qualifié par les caractères de globalité (grand récit d'émancipation), d'étatisme (rôle de la puissance publique) et d'optimisme (foi dans le progrès et la science). Il s'est manifesté sous deux formes, attestataire (conformité aux valeurs dominantes), et surtout contestataire (rupture avec la société et les valeurs dominantes, comme pour certains courants architecturaux du Mouvement moderne, ou pour le constructivisme russe et son slogan «changer la ville, changer la vie »: le projet spatial s'articule à un projet social.)

Avec les mutations évoquées de la société post-industrielle, le projet moderne change aussi de nature. La crise de la modernité, la montée de l'individualisme, l'extension du localisme, les revendications identitaires, et surtout la critique du progrès et la perte de foi dans la science et la technique, caractéristiques de cette situation de postmodernité ${ }^{18}$, vont affecter directement la signification du projet et sa nature : il va devenir local, individualisé, particulier à des groupes, tout en se démocratisant (on parle de démocratie locale, associative, participative). Il perd surtout son caractère optimiste et contestataire pour devenir essentiellement attestataire (conservation de l'ordre établi). N'étant plus global, la question qui se pose maintenant, pour lui, est la coordination de la pluralité des petits projets particuliers (v. le projet d'intercommunalité, par exemple).

Cette diffusion de la notion de projet est confirmée par d'autres chercheurs comme Chr. Boltansky ${ }^{19}$, pour qui le capitalisme s'est profondément modifié ces trente dernières années générant un nouveau modèle d'entreprise, de nouvelles formes d'encadrement du travail salarié, des nouvelle normes de production : ces transformations, dit-il, se sont faites principalement autour de dispositifs comme le réseau et le projet. Pour l'auteur la notion est en train de déborder le monde du travail pour envahir la vie privée et affective : on assiste à la mise en place d'une organisation générale de la société fondée sur le projet.

\footnotetext{
${ }^{15}$ J. P. Boutinet, Anthropologie du projet, Paris, Puf, 1993.

${ }^{16}$ F. Choay, La règle et le modèle. Sur la théorie de l'architecture et de l'urbanisme, Paris, Le Seuil, 1980.

${ }^{17}$ La Révolution voulait construire une nouvelle société, mais il faudra, on le sait, attendre la IIIe République (1885) pour voir le début de la réalisation de ce projet politique : une société démocratique. Pour F. Furet, la Révolution se terminait avec la IIIe République,

18 J. F. Lyotard, La condition post-moderne, Paris, Minuit, 1979 ; Le post-moderne expliqué aux enfants, Paris, Galilée, 1986

${ }^{19}$ Chr. Boltansky, E. Chapello, Le nouvel esprit du capitalisme, Paris, Gallimard, 1999
} 
Cette généralisation, cette diffusion, ces caractéristiques liées à la postmodernité définissent la nouvelle culture du projet qui va se répercuter également sur le projet urbain : projet de ville, projet de territoire, projet stratégique, projet local..., il se répand dans la pratique urbanistique pour désigner la nouvelle façon dont une collectivité territoriale se saisit de son espace pour concevoir et organiser son développement dans le temps, mais en cherchant plus à s'adapter à l'économie mondialisée qu'à la remettre en question ${ }^{20}$.

\section{Une complexification du système d'acteurs du projet urbain}

Le deuxième aspect a trait à la complexification du système d'acteurs et du système de décision qui lui est lié. Depuis 1982, avec la décentralisation, l'urbanisme a cessé d'être le monopole de l'Etat, sa compétence exclusive, et s'est déplacé au niveau local, engendrant et mobilisant une multiplicité d'acteurs (élus, maîtres d'ouvrage, maîtres d'œuvre, experts, habitants...) et de nouveaux métiers, qui impliquent un système de décision de plus en plus lourd avec des interactions de plus en plus nombreuses et complexes.

Y. Janvier ${ }^{21}$ a étudié les mutations de ce système d'acteurs et du dispositif de conduite du projet urbain. Le modèle de management étatique, hiérarchique, autoritaire et sectoriel est fini : de nouvelles démarches, de nouveaux acteurs, de nouveaux métiers interviennent, à présent, tout au long du processus de projet. Le schéma linéaire classique de production de l'espace, programmation - conception - réalisation, est dépassé, une nouvelle organisation interactive plus complexe a vu le jour: elle est constituée de 4 grandes fonctions qui regroupent des acteurs précis et distincts, dans lesquelles les associations d'habitants et d'usagers sont de plus en plus impliquées (mais on reste encore loin de la co-décision). Pour son efficacité, ce nouveau système de production du projet doit rester ouvert, souple et flexible, adaptable à la conjoncture de plus en plus incertaine, donc révisable à tout moment. On résume ces fonctions.

-Une fonction de définition stratégique. C'est la fonction de définition de la politique urbaine et des grandes orientations stratégiques, de programmation et de conception du projet de ville, mais également d'élaboration du parti (spatial) d'aménagement (interviennent ici, outre les élus, les experts, les agences d'urbanisme...)

-Une fonction de portage politique. C'est la fonction de prise en charge financière et de responsabilité politique assumée sur le long terme par les élus, institutions, et autres maîtres d'ouvrage, qui portent le projet urbain dans le temps, en assurant sa continuité, et en procédant périodiquement aussi à son évaluation.

-Une fonction de pilotage technique. C'est la fonction de conduite et de coordination du projet par un comité de pilotage qui joue le rôle de «chef d'orchestre». Située à l'intersection de la fonction de portage et de la fonction de réalisation, elle doit traduire sur le terrain les principaux choix stratégiques effectués en diverses opérations particulières, contrôler la cohérence globale du projet, et surtout garantir sa faisabilité économique (les principaux acteurs ici sont les aménageurs, en partenariat mixte privé-public, SEM, EPA...)

\footnotetext{
${ }^{20} \mathrm{~F}$. Choay voit cependant encore dans la proposition de A. Magnaghi, Le projet local, Spirmont, Mardaga, 2003, une survivance de l'utopie concrète en urbanisme, « une alternative stratégique au développement global », L'utopie et le statut anthropologique de l'espace édifié, Esprit, 10, octobre 2005.

${ }^{21}$ Y. Janvier, Un système de production en mutation, in A. Masboungi (éd), Fabriquer la ville, Outils et méthodes: les aménageurs proposent, Paris, La Documentation Française, 2001 ; L'Avenir des structures d'aménagement, rapport de recherche Club Ville Aménagement, Juin 1999.
} 
-Une fonction de réalisation. C'est l'opération finale de réalisation et de concrétisation sur le terrain des objectifs préalablement définis (interviennent ici les opérateurs, développeurs, promoteurs, architectes d'opération...)

Ces 4 fonctions, avec leurs acteurs spécifiques, sont nécessaires à tout projet urbain, ils ne doivent pas être confondues avec les phases chronologiques de déroulement du projet. La vieille linéarité du projet (amont/aval) fait place maintenant à une interaction de ces fonctions (feed back). Dès l'amont du projet (phase stratégique) la co-conception (travail collectif) entre acteurs est la règle. L'articulation portage politique / pilotage technique est le point sensible de tout le système de production et détermine la qualité du projet. Telles sont, rapidement décrites, les principales mutations du mode de production et du système d'acteurs, et les grandes fonctions, qui caractérisent le système actuel de production du projet.

Le projet PRG peut être analysé, dans son déroulement, en trois grandes phases successives et interactives, amont/intermédiaire/aval, où se retrouvent ces 4 fonctions.

a) Phase amont du projet. C'est là qu'intervient la fonction stratégique. La conception est élaborée et révisée tout au long du processus, tant dans la programmation (arrivée de l'université dans la ZAC, par exemple) que dans la forme urbaine (le parti d'aménagement, œuvre de l'APUR, est aussi sans cesse revu et corrigé). Des modifications, en rapport avec les 3 dates citées (1989 arrivée de la BNF, 1997 installation de la concertation, 2001 conquête du pouvoir municipal par la gauche) vont agir sur le mécanisme de décision (concertation et rôle de la Ville) avec des impacts sur le projet.

b) Phase intermédiaire du projet. Se combinent ici les fonctions de portage politique et de pilotage technique assurées par La SEMAPA qui entre en scène en 1991. Elle va pratiquer à partir de 1997 - cas unique à Paris - la concertation permanente avec les associations de l'arrondissement. Le travail consiste surtout, dans cette phase, à élaborer les dossiers de concours et à organiser les consultations pour choisir les futurs architectes coordonnateurs, responsables des différents secteurs. La diversité des architectes et la variété des solutions urbaines par secteurs recherchées sont destinées à éviter la monotonie et la standardisation, critère essentiel de qualité urbaine pour l'aménageur.

c) Phase aval du projet. C'est la fonction de réalisation finale par des opérations particulières sur des parcelles équipées et vendues par l'aménageur qui se déroulent avec des acteurs spécifiques, selon les secteurs, sous la direction d'architectes coordonnateurs choisis sur concours, et sous le contrôle de la SEMAPA (exemple pour un lot dans le Secteur Austerlitz: architecte coordonnateur $=\mathrm{Chr}$. Devillers $;$ promoteur $=\mathrm{SCIC}$; utilisateur $=$ Caisse des dépôts $;$ architecte d'opération $=$ Chr. Hauvette).

On a donc, à la fois, dans cette structuration générale du projet urbain, une division territoriale de la ZAC en secteurs, et une " sédimentation projectuelle » (superposition) d'acteurs successifs (Ville/ SEMAPA/ archi. coordonnateurs/ archi. opérationnels/utilisateurs ${ }^{22}$ ) pour pouvoir obtenir la diversité et la variété recherchées.

On voit de quelle manière le projet urbain, qui s'étire sur une longue période, mobilise, au cours de ses différentes phases d'actualisation, des acteurs nombreux, chaque fois distincts, selon les phases du projet et les différents fonctions de l'aménagement, tout en conservant des rapports interactifs entre elles, la vielle linéarité conception - réalisation étant rompue : le projet doit rester souple pour s'adapter à la conjoncture. Enfin, loin d'être un projet contestataire, ou même innovant dans ses formes, le projet PRG cherche, avant tout, à intégrer Paris au nouveau contexte métropolitain en renforçant sa position centrale, et à la mondialisation (compétition urbaine), tout en s'adaptant aux fluctuations du marché immobilier parisien.

\footnotetext{
${ }^{22}$ D'autres acteurs sont également présents dans ce projet urbain : SNCF, APHP, Etat, Région, RATP.....
} 


\section{L'irruption et l'essor du débat public dans l'aménagement}

Le troisième point porte sur le monopole des experts et des élus qui est aujourd'hui remis en cause : partout, sur toutes les grandes questions de société et d'aménagement, tant à l'échelle locale que nationale (TGV, école, OGM, nouvel aéroport, autoroutes...) le débat public est introduit. Son apparition est aussi liée à la crise générale de la représentation politique ${ }^{23}$, à la critique de la centralisation, à une demande plus grande de démocratie locale, de démocratie de proximité. On peut rappeler quelques dates : 1983, loi Bouchardeau sur la démocratisation de l'enquête publique, en aval ; 1992, loi Bianco sur la démocratisation de l'enquête publique, plus en amont ; 1995, création de la CNDP (Commission nationale du débat publique) sur le modèle du BAPE québécois (Bureau des audiences publiques sur l'environnement, 1978) ; 1996, loi Lepage (Charte de la concertation en matière d'environnement) ; 2001, loi sur la démocratie de proximité (sur les conseils de quartier)...

M. Callon, P. Lascoumes, Y. Barthe ${ }^{24}$ ont analysé ces nouveaux lieux de débat en utilisant le concept de «forum hybride» qui combine savoir profane et savoir savant, recherche de laboratoire et recherche de plein air. Ils ont fait également une classification de ces forums : selon eux, la conférence citoyenne reste la meilleure structure de débat public, par la participation des non-spécialistes qu'elle permet, par la capacité qu'elle offre à de nouveaux groupes de pouvoir s'exprimer, par la qualité des discussions obtenue.

Mais le débat public, la concertation, la participation, suscitent de nombreuses réserves chez un grand nombre de chercheurs qui parlent de «poudre aux yeux ». Les critiques portent sur la représentativité des délégués (souvent des « notables »), sur la nature de la décision (la concertation n'est qu'une simple consultation pour avis), sur la transparence des débats (les profanes sont noyés sous un flot d'informations), sur la difficulté du débat (cacophonie), et surtout sur le syndrome Nimby $^{25}$ (défense égoïste des petits intérêts particuliers, locaux, au détriment de l'intérêt général). Néanmoins, malgré ces difficultés, et même si l'on est encore loin de la véritable co-décision, l'introduction du débat s'élargit de plus en plus et reste globalement positive : la question de l'expertise citoyenne est, de plus en plus, à l'ordre du jour.

A Paris Rive Gauche, la concertation est introduite dès 1997 avec l'application de la loi Lepage $^{26}$. On peut résumer les principes de cette charte : la concertation commence en amont, elle est aussi large que possible, elle est mise en œuvre par les pouvoirs publics, elle exige la transparence, elle s'organise autour de points forts (opportunité du projet, définition du projet, réalisation du projet), elle nécessite la présence d'un Garant, elle est financée par le maître d'ouvrage, elle fait l'objet d'un bilan (annuel). D'une façon générale, elle vise «un enrichissement de la démocratie représentative par une démocratie participative ».

La SEMAPA en fera une traduction fidèle: une douzaine d'associations y participent au départ réunies en Bureau des associations avec un Chargé de mission élu, rémunéré par l'aménageur, et un Garant (personnalité extérieure qui contrôle le déroulement et la conformité des débats à la charte). Deux modalités principales de travail sont prévues: le Comité permanent de concertation (CPC) qui se réunit tous les trois mois, avec une certaine souplesse, et en séance

\footnotetext{
${ }^{23}$ Sur cette question, Collectif, La démocratie locale, représentation, participation, et espace public, CRAPS/ CURAPP, Paris, Puf, 1999. A. Levy, La démocratie locale en France : enjeux et obstacles, Espaces et Sociétés, Ville et démocratie, 112, Paris, L'Harmattan, 2004.

${ }^{24}$ M. Callon, P. Lascoumes, Y. Barthe, Agir dans un monde incertain, Paris, Le Seuil, 2001

${ }^{25}$ Not in my back yard.

${ }^{26}$ Charte de la concertation, ministère de l'Aménagement du Territoire et de l'Environnement (5.07.96). Suite au projet de rénovation des Halles et à la polémique soulevée par ce concours, les associations concernées ont également adopté cette charte pour structurer le débat public sur le futur du quartier.
} 
publique annuelle pour le bilan, des Groupes de travail thématique, plus restreints, plus fréquents, sur tel ou tel sujet d'aménagement précis. C'est la Ville de Paris, à travers son chef de projet à la Direction de l'Urbanisme, qui assure la direction des débats.

Outre ces dispositifs, la mairie du XIIIe arrondissement a ajouté les Samedis de la concertation (2001) convoqués sur des questions spécifiques (Université, transport...). Enfin, avec la loi sur la démocratie de proximité, trois conseils de quartier ont été créés sur l'ensemble des secteurs où sont discutés des problèmes spécifiques au quartier. On peut se demander si tous ces dispositifs de négociation ne font pas double emploi (?). Toute cette démarche vise finalement, comme dit un élu, à rompre avec le "schéma habituel de la confrontation citoyens / aménageurs 》: la démocratie participative c'est, en effet, la recherche d'une pacification des rapports entre aménageurs, experts, élus et citoyens ${ }^{27}$, en fabricant du consensus, mais aussi en explicitant les dissensions.

On voit bien ici les changements opérés dans les mécanismes de décision et les transformations des anciennes pratiques induites par cette ouverture du projet au débat public, par cette volonté de démocratisation de la décision; mais elle reste encore, et en dernier ressort, souvent entre les mains des élus et des experts.

Quels sont les impacts que ces transformations vont avoir sur l'évolution de l'urbanisme et sa pratique?

\section{Vers un urbanisme transactionnel ?}

Outre le vide doctrinal évoqué caractérisant l'urbanisme actuel, on a vu qu'un nouveau contexte conditionnant sa pratique a vu le jour : une nouvelle culture de projet devenu attestataire, éclaté en différents petits projets particuliers, locaux..., à coordonner; une complexification du système d'acteurs avec multiplication de représentations antagonistes sur la ville, sa forme, entraînant une confrontation entre idées de ville opposées et objectifs distincts..., à arbitrer ; une extension du débat public dans l'aménagement, introduisant dans le processus de décision le citoyen (habitant et usager) avec ses préoccupations spécifiques et ses désirs..., à débattre. Ces nouvelles conditions de production donnent lieu à une nouvelle pratique qu'on propose d'appeler urbanisme transactionnel.

Organiser les échanges entre acteurs, coordonner les actions, rechercher des compromis pratiques entre positions et visions adverses, arbitrer des situations de conflit, débattre avec les habitants, telles sont les taches principales de cet urbanisme transactionnel. Ces transactions, d'où résultent la forme et le contenu du projet final, peuvent se dérouler de deux manières: soit polémique, c'est-à-dire par des rapports de force entre acteurs (action judiciaire, manifestation, occupation de locaux...), soit contractuelle, dans le cadre de structures de négociation préétablies (comité, conseil, commission...), par dialogue et débat (souvent pour éviter la première modalité conflictuelle).

Ce nouvel urbanisme transactionnel, que d'autres appellent aussi «urbanisme concourrant $»^{28}$, veut répondre à la question posée par la production collective de l'espace, ou co-production du projet. Urbanisme de négociation, il relève également de la problématique contemporaine de la gouvernance ${ }^{29}$ : ces transactions se structurent à la fois horizontalement (co-conception, co-décision entre acteurs, entre partenaires privés et publics) et verticalement (interventions d'acteurs par strates successives, du plus général au particulier). C'est enfin un

\footnotetext{
${ }^{27}$ Rappelons que, suite à l'action judiciaire contre le PAZ de 1990 menée par les écologistes, le plan est annulé par le tribunal administratif le 11/03/93, pour insuffisance d'espace vert, bloquant ainsi le projet PRG.

${ }^{28} \mathrm{~F}$. Ascher, Les nouveaux principes de l'urbanisme ..., op.cit.

${ }^{29}$ Sur cette question, par exemple, Les Annales de la recherche urbaine, Gouvernances, 80-81, dec. 1998.
} 
urbanisme procédural qui nécessite pour fonctionner des règles de droit (loi Deferre sur la décentralisation, loi Chevènement sur l'intercommunalité, loi Voynet sur les Pays, loi SRU sur la mixité et la participation...) avec lesquelles il tend à se confondre. Plus d'objectif de transformation sociale, le «projet » qui le caractérise est, on l'a dit, purement attestataire : l'utopie ${ }^{30}$ a cessé d'être le ressort de l'urbanisme occidental, il est gagné par l'air du temps, le nouvel esprit gestionnaire ${ }^{31}$ et la nouvelle idéologie managériale qui l'accompagne, assimilant la ville à une entreprise et son maire à un manager, avec pour critères dominants le rendement (fiscal) et le profit (plus-value immobilière).

Transactionnel, procédural, gestionnaire, tels sont les caractères de ce nouvel urbanisme qui correspondent à sa désubstantialisation. Devenu amnésique de son histoire, il semble avoir oublié l'identité de la discipline d'où il est issu ${ }^{32}$. Dénué de toute préoccupation d'innovation spatiale et sociale, sans modèle global, ni orientation particulière, il est ouvert à tous les possibles, à toutes les opportunités. Le vide doctrinal de la situation actuelle évoqué (caractéristique de la postmodernité) est un autre signe de cette désubstantialisation: l'urbanisme contemporain n'est plus qu'une affaire d'arrangements entre acteurs ${ }^{33}$, au statut souvent inégal, le produit de transactions entre leurs visions et leurs intérêts particuliers, à un moment donné, dans un lieu donné.

A Paris Rive Gauche, entre le projet initial de départ et le résultat final à l'arrivée, les multiples changements du projet survenus résultent de transactions successives entre les acteurs, par coopération ou confrontation entre les idées, représentations et stratégies des protagonistes. La SNCF va, par exemple, pour ses propres raisons stratégiques, différer de 15 ans la réhabilitation de la gare d'Austerlitz qui vient à peine de démarrer, alors que le reste de la ZAC est déjà bien avancé, soulevant des problèmes aujourd'hui pour l'articulation de ces deux grandes parties du projet urbain pensées séparément (l'aile du buffet de la gare est, par exemple, menacée par la construction de la dalle). Le parti d'aménagement (un urbanisme de dalle recouvrant les voies ferrées, mais voulu différent de celui de la Défense) est élaboré par l'APUR après une série de consultations étalées sur prés de 10 ans (1983-1990-1993) : la conception de la forme du tissu, conformément à la doctrine de l'APUR, est d'inspiration néo-haussmannienne, c'est-à-dire une forme urbaine traditionnelle constituée d'îlots et de rues qui prolongent le tissu du XIIIe jusqu'à la Seine, mais qui reste, paradoxalement, sans composition réelle : les îlots, alignés sur rue, sont simplement juxtaposés latéralement à la $\mathrm{BNF}^{34}$. Portzamparc y introduit dans son secteur (Tolbiac) son principe de l'îlot ouvert, mais les îlots existants, trop petits, posent des problèmes pour son application (vis-à-vis et promiscuité). Le tracé de l'avenue de France, linéaire (sur 2 $\mathrm{km}$ ) et sans rupture (une place), ni articulation spatiale aux extrémités (périphérique et gare), divise la ZAC en coté nord et sud. L'ensemble du projet semble avoir été conçu sans une centralité prédéfinie (absence de programmation précise des commerces et des loisirs).

Le programme de départ, une forte densité de logements et surtout de bureaux, devait équilibrer l'Est par rapport à l'Ouest parisien. Le PAZ (1990) fut attaqué en justice par les associations

\footnotetext{
${ }^{30}$ C'est ce que pense aussi F. Choay, L'utopie et le statut... op.cit., qui voit, comme on l'a dit, dans les propositions d'A. Magnaghi, op.cit., une nouvelle version du modèle " utopie concrète ».

${ }^{31} \mathrm{~V}$. Claude, Faire la ville. Les métiers de l'urbanisme au XXème siècle, Marseille, Parenthèses, 2006, constate également cette évolution de l'urbanisme vers «l'air du temps, l'attitude gestionnaire », mais sans tirer les conclusions de cette observation.

${ }^{32}$ Cela est confirmé aussi par la marginalisation, voire la quasi disparition de l'enseignement de l'histoire de l'urbanisme dans les écoles d'architecture et dans les Instituts d'urbanisme. Il en va de même pour l'enseignement de la typo-morphologie qui sensibilisait les étudiants à l'espace urbain, à ses formes et à ses structures, qui tend aussi à reculer, après avoir eu ses heures de gloire dans les années 70-80.

${ }^{33}$ Avec la figure du maire devenu « premier urbaniste ».

${ }^{34}$ Le caractère d'espace public de cette esplanade surélevée, avec son jardin inférieur inaccessible, qui voudrait, selon son architecte, poursuivre l'aménagement des grands espaces libres le long de la Seine (Champ de Mars, Invalides, place de la Concorde...) est discutable : il fonctionne comme un gigantesque perron d'accès à la $\mathrm{BN}$.
} 
pour insuffisance d'espace vert et trop forte densité, et annulé. De même, pour le sort réservé à la gare dans le plan de l'APUR : la halle (pourtant classée) était percée par une voie reliant les deux rives, les deux ailes de la gare et le bâtiment de tête étaient également rasés par le tracé de l'avenue de France qui débouchait directement sur la place Valhubert... Tout ce secteur Austerlitz sera révisé sous la pression des associations, obligeant le tracé de l'avenue à éviter la gare en tournant brutalement sur le quai, préservant ainsi la halle et le bâtiment de tête (vendu par la SNCF, il est converti en bureaux par une triste opération de façadisme).

Pour prévenir tout nouveau conflit, la SEMAPA installe la concertation en 1997 et institutionnalise le débat. La conservation et la requalification des bâtiments anciens du site (Frigos, Grands Moulins, usine Sudac...), presque tous rasés dans le projet initial de l'APUR, sont à mettre à l'actif des associations ${ }^{35}$, il en va de même pour la lutte actuelle qu'elles mènent concernant la conservation (intégrale) de la halle Surnam, du buffet de la gare, des actions menées ça et là pour baisser les gabarits de certaines constructions, contre l'implantation du TGI à Tolbiac (tertiarisation excessive du quartier), pour l'extension des espaces verts...

L'arrivée de l'Université Paris VII avec ses labos de recherche, l'accroissement du logement social, la baisse de densité des bureaux - liée également à la crise de l'immobilier (le tertiaire sera surtout de nature publique ou parapublique) - sont les changements majeurs survenus dans la programmation, défendus par la nouvelle municipalité et soutenus par les associations qui militent pour plus de mixité. Une kyrielle d'architectes-coordonnateurs ${ }^{36}$ va intervenir, chacun avec ses idées propres et ses visions personnelles, parfois opposées, suite aux multiples concours organisés (Andreu, Schweitzer, Portzamparc, Devillers, Fortier, Reichen, Lion, Gangnet, Jacob \& MacFarlan ...), auxquels il faut ajouter aussi les divers architectes d'opérations particulières, apportant chacun leur touche personnelle. Une ébauche de centralité est finalement esquissée avec l'arrivée du complexe de loisirs MK2, sur une parcelle triangulaire résiduelle, non prévue à cet usage, face à la BNF.

Toutes ces transformations et réalisations successives sont effectuées à partir de confrontation entre idées et représentations opposées des différents acteurs, tout au long du projet, impliquant négociations et co-conceptions, justifiant le qualificatif transactionnel. L'objectif de cette diversité recherchée, par ces multiples interventions et transactions, est, pour la SEMAPA, de produire la complexité souhaitée, synonyme, pour elle, de "sédimentation projectuelle ", afin, de mimer le processus de croissance des villes anciennes.

Cet urbanisme transactionnel présuppose, au départ, un pluralisme de représentations urbaines liées aux différents acteurs, à leurs conceptions de la « ville bonne », à partir desquelles compromis et synthèses sont construits par négociation. Mais on pourrait aussi, à l'inverse, poser ce pluralisme des idées et des positions, non comme une contrainte initiale, mais comme un objectif final à atteindre : plus que la décision, le débat démocratique ${ }^{37}$

\footnotetext{
35 Parmi les nombreuses associations du XIIIe arrondissement qui défendent chacune des intérêts particuliers (piétons, locataires, paysage...), spatialement circonscrits (immeuble, rue, quartier...), les Verts (Ecologie pour Paris) sont les seuls qui proposent une vision urbaine plus large et structurée, synthétisée dans un manifeste, Paris Rive Gauche, Le projet des Verts, Paris, Presses Pluriel, 2001. SOS Paris, tout en couvrant la capitale, se donne comme objectif principal la défense du patrimoine historique. Il faut signaler aussi TAM TAM, une des plus anciennes et des plus actives associations de l'arrondissement, ADA 13 ...

${ }^{36}$ L'apport de ces architectes-urbanistes consiste surtout dans 1'« urban design» (composition urbaine) du secteur concerné.

${ }^{37}$ Ce débat renvoie aussi au débat politique plus général entre démocratie procédurale (J. Rawls) et démocratie communautariste (M. Sandel, M. Walzer), plus substantielle : là-dessus, Ph. de Lara, L'intérêt général entre justice et communauté, in $\mathrm{Ph}$. Genestier (éd), op. cit; également $\mathrm{F}$. Ascher, De l'intérêt général substantiel à l'intérêt général procédural ?, Ibid. L'auteur y oppose l'intérêt général substantiel construit en France par l'Etat à l'intérêt général procédural construit dans les pays anglo-saxons «comme somme consensuelle des intérêts particuliers », qu'il préfère. Nous vivons aujourd'hui, dit-il, « la fin du prêt-à-porter idéologique », mais le pragmatisme qu'il défend n'est-il pas la nouvelle idéologie de notre temps ? Le 'laissez-faire, laissez-aller' des intérêts particuliers ne définit-il pas le libéralisme?
} 
consisterait alors plutôt à organiser la réflexion sur la production de pratiques et d'idées urbanistiques nouvelles...

Entre l'urbanisme fonctionnaliste substantiel de la période précédente, le Mouvement moderne avec ses modèles formels (CIAM, Cité-jardin...), critiqué, à juste titre, pour son réductionnisme, sa prétention à l'universalité, son autoritarisme, et l'urbanisme gestionnaire désubstantialisé de la période actuelle qui se veut plus démocratique - mais qui conserve encore, en réalité, une hiérarchie dans le système de décision - ne faudrait-il pas chercher une voie médiane, en réinscrivant l'évolution de l'urbanisme à l'intérieur de l'histoire de la discipline, en réinterrogant ses finalités politiques (dans une société démocratique), l'espace et ses significations, et surtout en ne perdant pas de vue l'articulation essentielle entre projet social et projet spatial ? Réinsuffler de la substance (sociale/spatiale) dans l'urbanisme, sans nier les acquis de la démarche procédurale, ni les avancées de sa démocratisation (à renforcer), tels sont, aussi, les défis à relever par la recherche urbanistique pour affronter la ville d'aujourd'hui, la ville de l'age post-industriel.

*Architecte, chercheur au CNRS, UMR 7136, Laboratoire Théorie des mutations urbaines, Institut Français d'Urbanisme, Université Paris VIII. Dernier ouvrage paru : Les machines à faire-croire, Formes et fonctionnements de la spatialité religieuse, Paris Anthropos/Economica, 2003. 